\title{
Effectiveness of Self-Compassion Training on Dysfunctional Attitudes and Quality of Life in Mine Explosion Amputees
}

\section{ART ICLE INF O}

\section{Article Type}

Original Research

\section{Authors}

Ghazanfari F. ${ }^{1} P h D$,

Shafiei M.*PhD

How to cite this article
Ghazanfari F, Shafiei M. Effective-
ness of Self-Compassion Training
on Dysf-unctional Attitudes and
Quality of Life in Mine Explosion
Amputees. Iranian Journal of War
\& Public Health. 2017;9(1):41-46.

*Psychology Department, Literature and Humanities Faculty, Lorestan University, Khorramabad, Iran ${ }^{1}$ Psychology Department, Literature and Humanities Faculty, Lorestan University, Khorramabad, Iran

\section{Correspondence}

Address: Central Organization of Lorestan University, Kilometer 5 of Tehran Road, Khorramabad, Iran. Postal Code: 68151-44316 Phone: +98 (66) 33234533

Fax: +98 (66) 33120627 masumeh.shafiei62@gmail.com

\section{Article History}

Received: October 31, 2016

Accepted: December 11, 2016

ePublished: March 10, 2017

\section{A B S T R A C T}

Aims Inefficient attitudes and low quality of life correlate with many behavioral disorders and problems. The aim of the study was to investigate the effects of self-compassion trainings on inefficient attitudes and quality of life in persons with amputations due to the land-mine explosions.

Materials \& Methods In the controlled pretest-posttest experimental study, 40 men with amputations due to the land-mine explosions, who were under the protection of the Martyrs Foundation of Gilan-E-Gharb Township, were studied in 2016. The subjects, selected via simple random sampling method, were randomly divided into two 20-person groups including experimental and control groups. Data was collected by the inefficient attitudes and quality of life questionnaires. Eight 1-hour compassion-training sessions were conducted in experimental group for two months, while control group received no intervention. Data was analyzed using multi-variable covariance analysis test.

Findings At the posttest stage, the pretest scores having been controlled, the mean scores of inefficient attitudes were significantly lower in experimental group compared to control group $(\mathrm{p}<0.01)$. In addition, the mean scores of quality of life and its components were significantly higher than control group $(\mathrm{p}<0.05)$.

Conclusion The self-compassion trainings improve the inefficient attitudes and quality of life in persons with amputations due to the land-mine explosions.

Keywords Compassion; Attitude; Quality of Life; Amputation

\section{CIT A T I O N L I N K S}

[1] A brief behavioral treatment of chronic post traumatic stress disorder in earthquake survivors ... [2] Long-term effects of war on psychosocial status of patients ... [3] Consideration of chronic pain and current prosthetic ... [4] Service satisfaction among war related ... [5] Development of adaptation questionnaire using ... [6] Diagnosis and statistical manual ... [7] Rumination and worry as mediators of the relationship ... [8] Self-compassion, self-regulation and ... [9] Relations among self-compassion, PTSD symptoms ... [10] Examining risk and resilience factors for depression ... [11] Selfcompassion in recovery following potentially traumatic ... [12] Fear of self- compassion and psychological inflexbility ... [13] Comparing self-compassion, mindfulness, ... [14] Measuring dysfunctional attitudes in the ... [15] Dysfunctional attitudes and the common mental ... [16] Evaluation of cognitive diathesis stress models ... [17] How may therapy and Practice of psychotherapy has ... [18] Post-traumatic growth of children affected ... [19] PTSD symptoms, satisfaction with life, and prejudicial ... [20] Peer support and outcome for veterans with ... [21] Quality of life and Quality of life and its influencing ... [22] Symptom benchmarks of improved quality ... [23] Spirituality, forgiveness, and quality ... [24] Study of health related quality of life in posttraumatic ... [25] Compassionate mind training for people ... [26] Contributions of dysfunctional attitude scale and general health ... [27] Factor analysis of the dysfunctional attitude ... [28] Effectiveness of cognitive-behavioral religious - cultural therapy in improvement of ... [29] The effect of exercise on quality of life of opioid dependent patients ... [30] Introducing comp-assion- focused ... [31] Self-Compassion: Conceptualizations, correlates \& int rventions review ... [32] Attachment and mental and physical health: Self-compassion and ... [33] Self-compassion in .. [34] Self -compassion, achievement goals, and cop ing with academic ... [35] Self-Compassion, Stress and ... [36] Cognitivebased compassion training: A promising prevention strategy for ... 


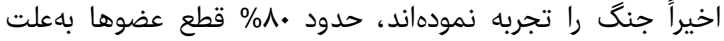

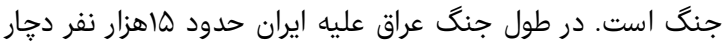

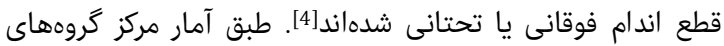

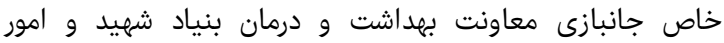

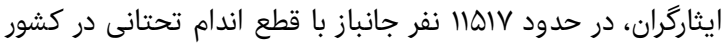

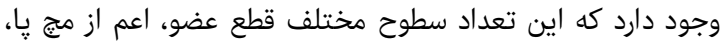

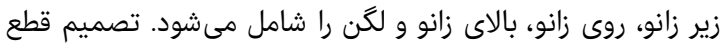

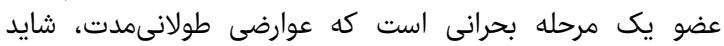

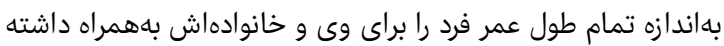

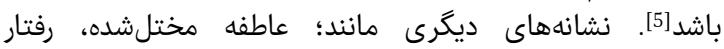

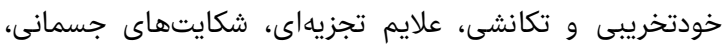

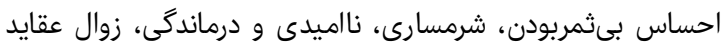

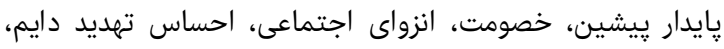

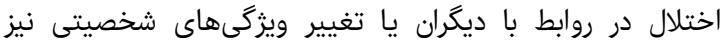

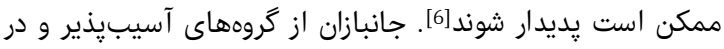

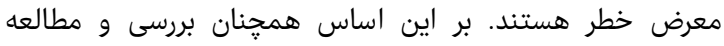

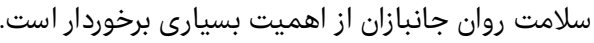

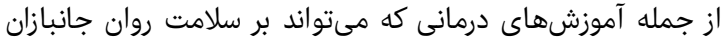

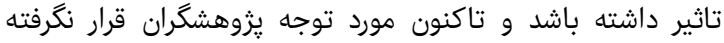

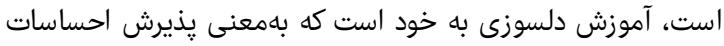

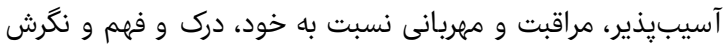

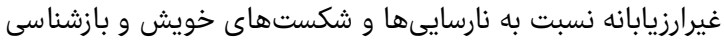

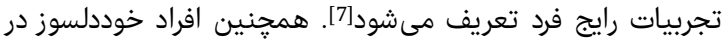

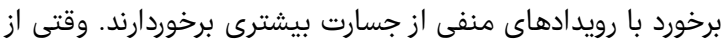

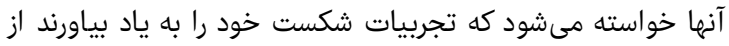

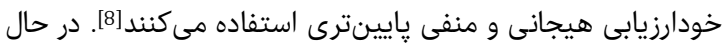

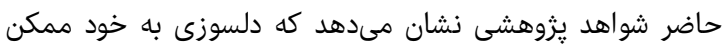

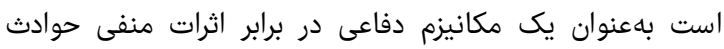

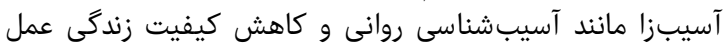

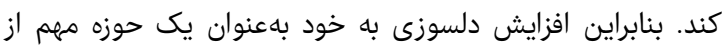

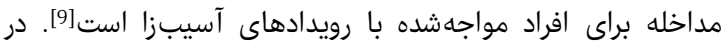

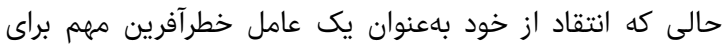

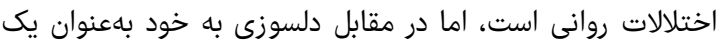

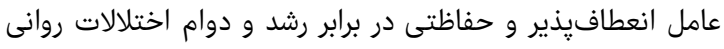

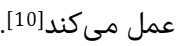

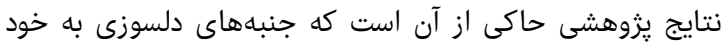

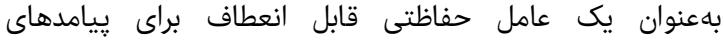

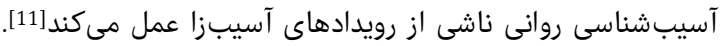

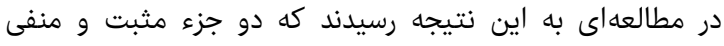

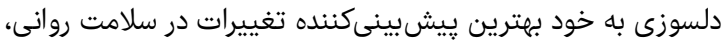

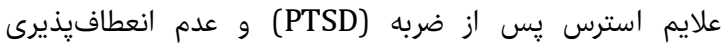

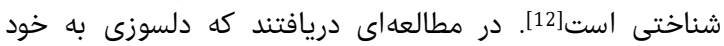

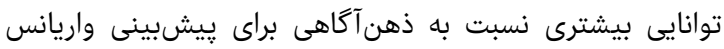

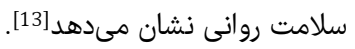

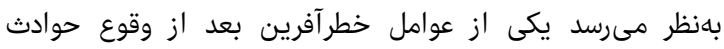

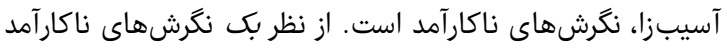

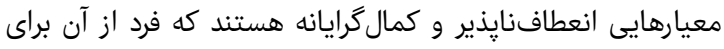

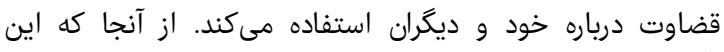

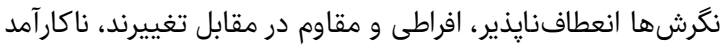

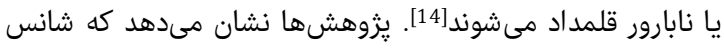

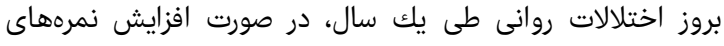

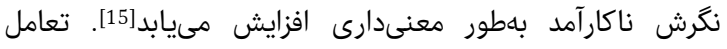

دوره 9، شماره ا، زمستان هوس1

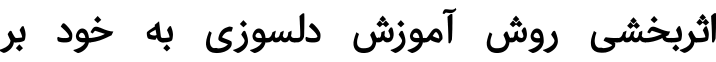

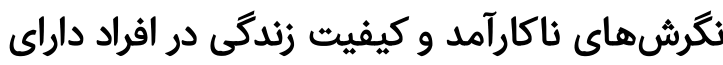 نقص عضو با انفجار مين}

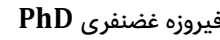
كَروه روانشناسى، دانشكده ادبيات و علوم انسانى، دانشكاه لرستان، خرمآباد، ايران

PhD " معصومه شفيعى ايرنى كروه روانشناسى، دانشكده ادبيات و علوم علوم انسانى، دانشگاه لرستان، خرمآباد، ايران

קكيده

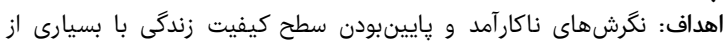

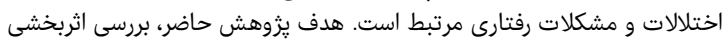

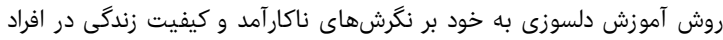

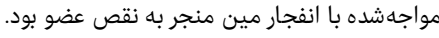

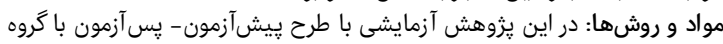

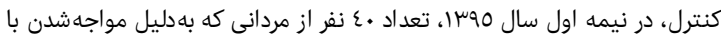

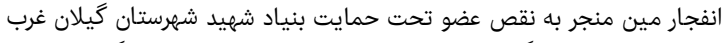

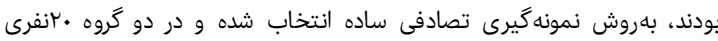

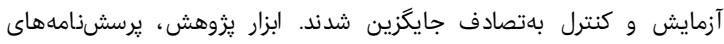

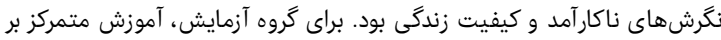

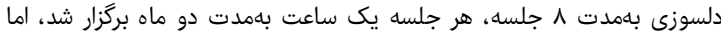

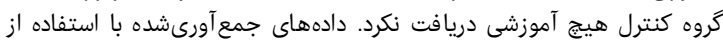

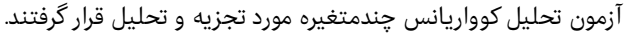

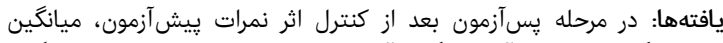

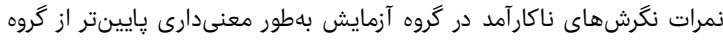

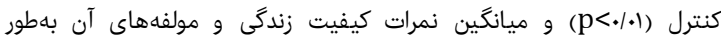

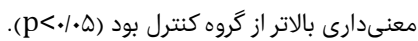

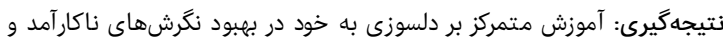

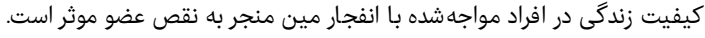

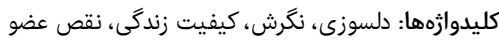

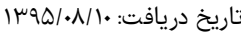

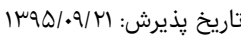

"نويسنده مسئول:

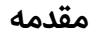

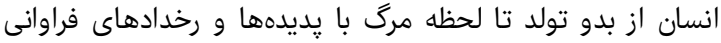

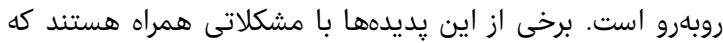

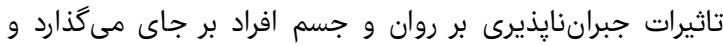

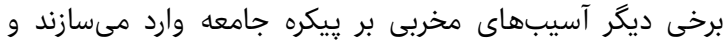

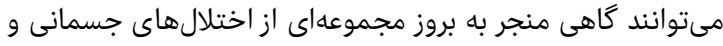

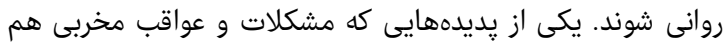

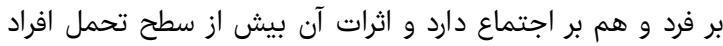

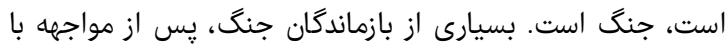

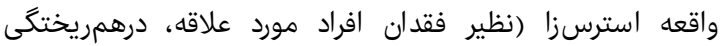

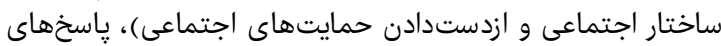

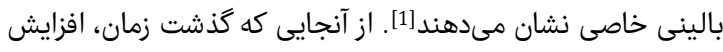

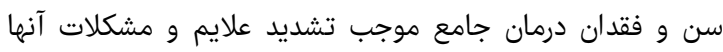

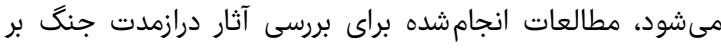

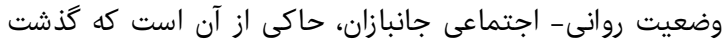

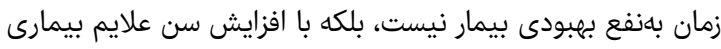

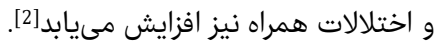

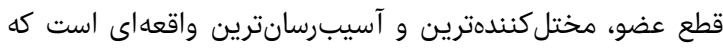
يك فرد ممكن است در زندگى خود تجربه كند [3]. در كشورهايى كه 
مواد و روشها

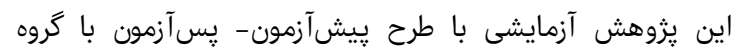

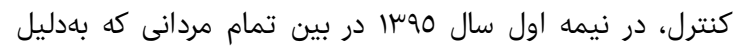

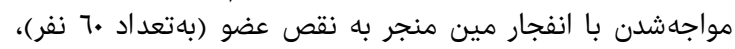

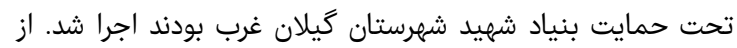

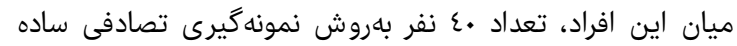

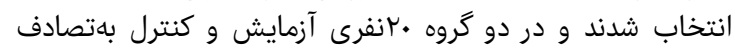

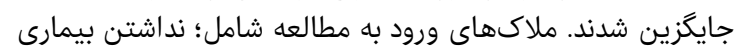

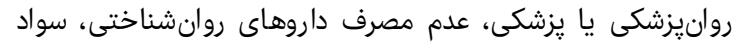

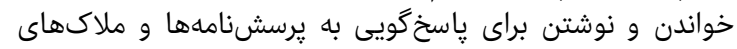

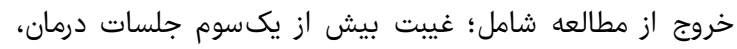

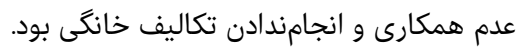

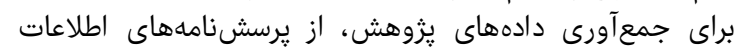
جمعيتشناختى، نكرشهاى ناكارآمد و كيفيت زندگى استفاده شد:

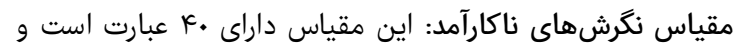

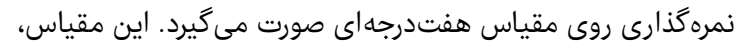

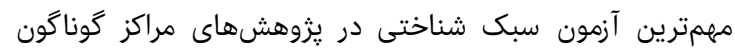

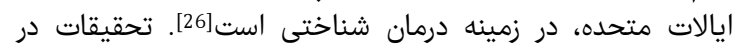

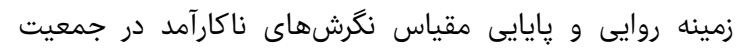

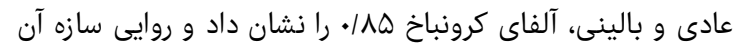

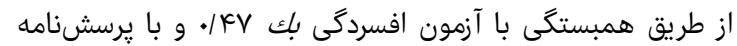

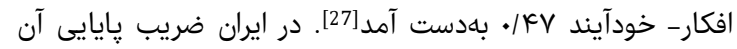

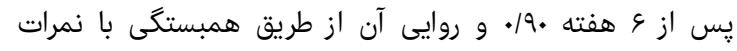

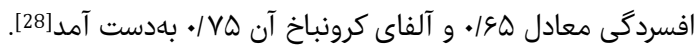

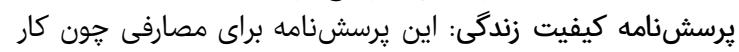

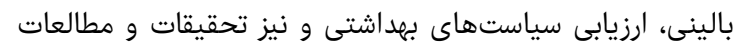

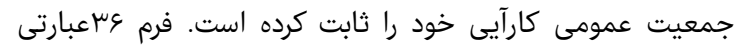

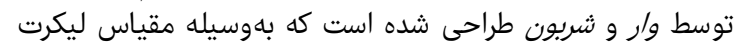

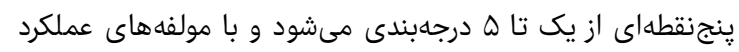

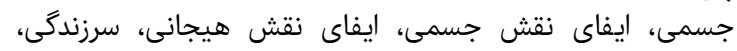

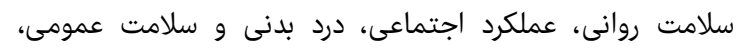

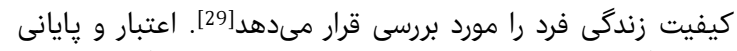

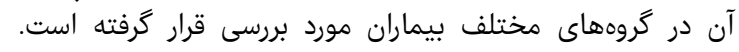

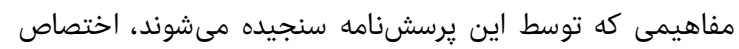

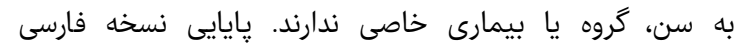

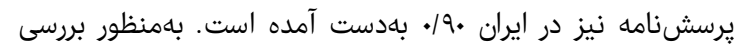

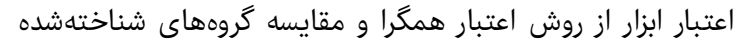

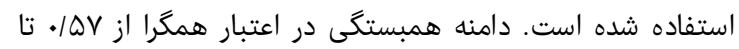

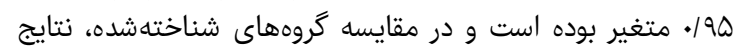
مورد بيشبينى در تمايز كيفيت زندكى بين مردان و زنان بهدست آمد [29]. آموزش متمركز بر دلسوزى، يكى از روشهاى رواندرمانى است كه

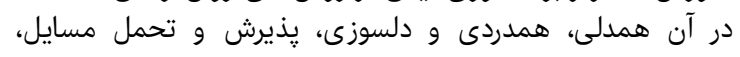

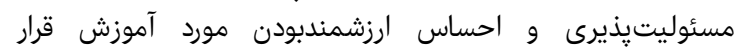

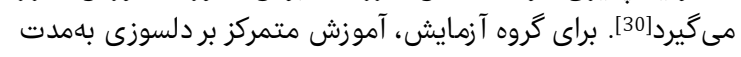

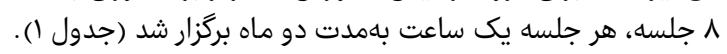

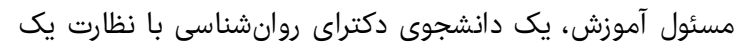

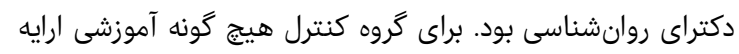

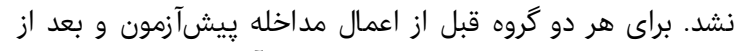

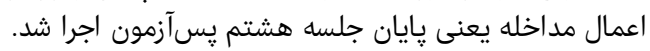

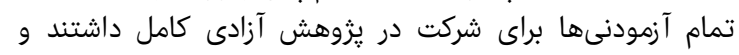

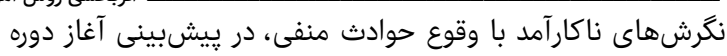

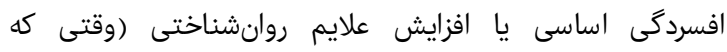

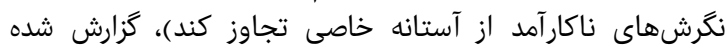

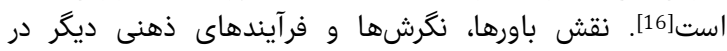

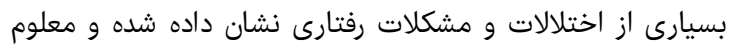

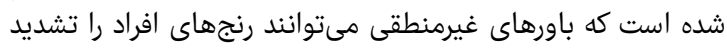

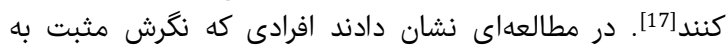

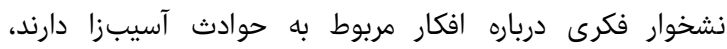

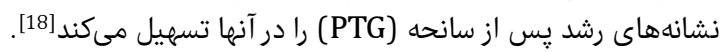

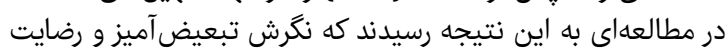

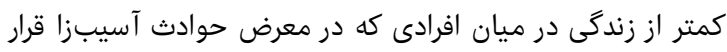

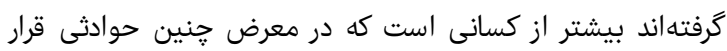

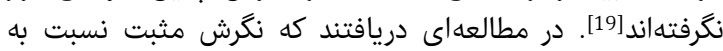

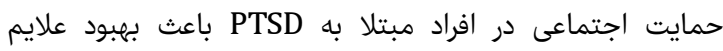
PTSD

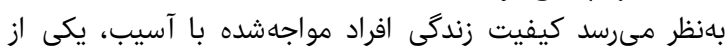

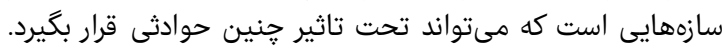

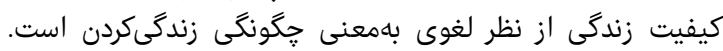

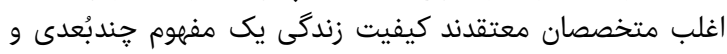

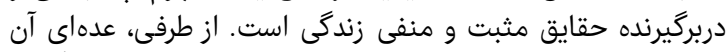

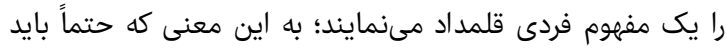

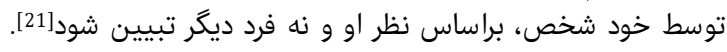

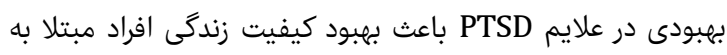
PTSD

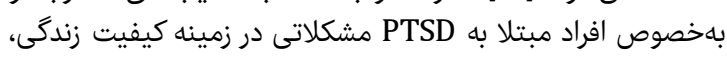

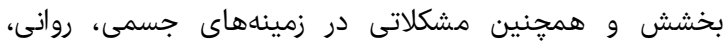

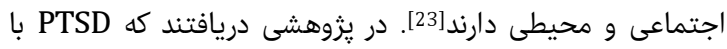

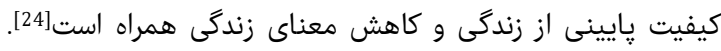

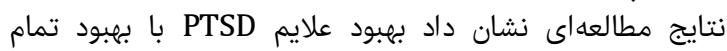

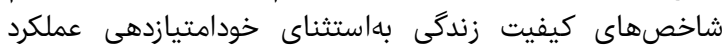

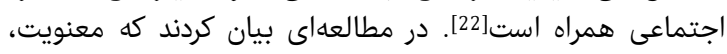

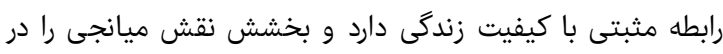
اين ارتباط داشت الشان.

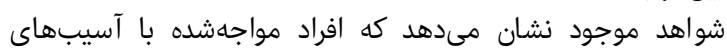

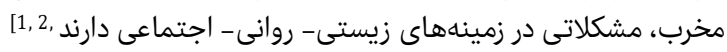

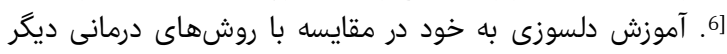

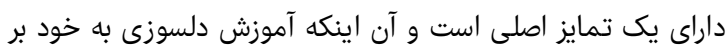

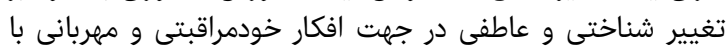

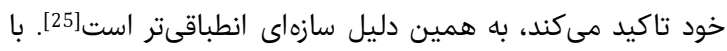

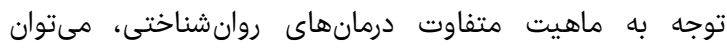

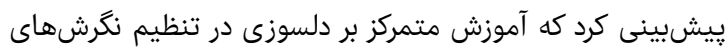

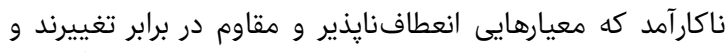

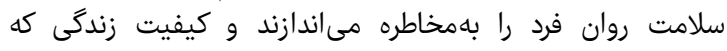

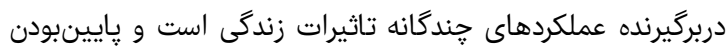

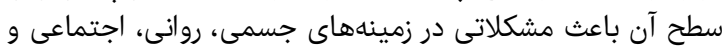

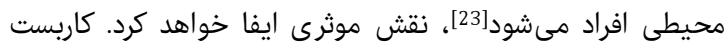

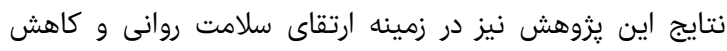

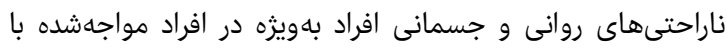

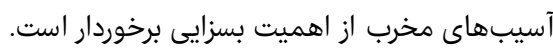

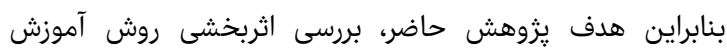

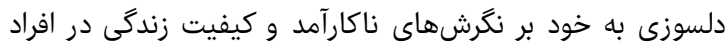

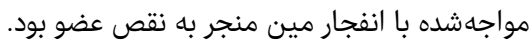


كيفيت زندگى و مولفههاى آن بهطور معنىدارى بالاتر از گروه

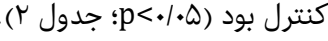

\begin{tabular}{|c|c|c|c|c|}
\hline ميزان تاثير & معنىدارى & 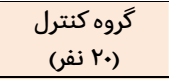 & 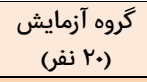 & متغيرها \\
\hline & & & \multicolumn{2}{|c|}{ نكرش هاى ناكارآمد } \\
\hline$\cdot 1 \cdot 1$ & . $18 \mathrm{r}$ & $\mid \& \wedge / 4 \cdot \pm 1 \cdot / \Lambda$. & $|V Y / \Lambda \cdot \pm I| / \Delta S$ & ي ي- آزمون \\
\hline \multirow[t]{2}{*}{.$|A|$} & $.1 . .1$ & $\left|F \Delta / V \mu_{ \pm}\right| R^{\mu} / R \Delta$ & $Q \Lambda / \Delta \mu \pm 1 . / 91$ & 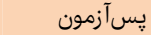 \\
\hline & $\cdot 1 \cdot V$ & $\mid r / K E \pm Y / 9 Y$ & $1.1 \varepsilon \xi \pm r /{ }^{\mu}$ & عملكرد جسمى آزمص. \\
\hline \multirow[t]{2}{*}{$\cdot|q|$} &. $\mid . .1$ & r & 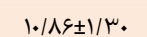 & زمون \\
\hline & & & \multicolumn{2}{|c|}{ ايفاى نقش جسمى } \\
\hline$\cdot 1 \cdot 1$ & $\cdot / \Delta \Lambda$ & $\mid r / K \varepsilon \pm r / \mu \Delta$. & $1 . / V^{\mu} \pm 1 / V_{q}$ & قآزمون \\
\hline \multirow[t]{2}{*}{. } & $.1 . r$ & $\mid \& / \Lambda \varepsilon \pm \Psi / q 9$ & $|9 / R| \pm|/ R|$ & 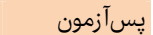 \\
\hline & & & \multicolumn{2}{|c|}{ ايفاى نقش هيجانى } \\
\hline س & .1 .9 & $\mid \mu / \psi \cdot \pm \mu / \cdot v$ & $11 / q \mu \pm r / \mu k$ & 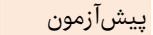 \\
\hline \multirow[t]{2}{*}{. } & .1 .1 & $\mid \psi / \varphi . \pm \mu / \varphi q$ & $|N / K \cdot \pm| / \Delta q$ & 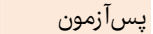 \\
\hline & & & & 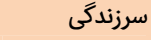 \\
\hline.$/$ &.$/ K F$ & $|r / F \varepsilon \pm \Psi / F|$ & $\| / \varepsilon \varepsilon \pm \Psi / 4 \mid$ & 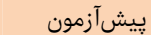 \\
\hline . $|\Delta|$ & $.1 . .1$ & $\mid r / Q H \pm r / Q Y$ & $\left|N / q \Psi_{ \pm} \pm 1 / q\right|$ & يَسآزمون \\
\hline$\cdot /$ & • & 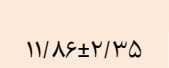 & $\mid r / F= \pm T / \Delta r$ & سيشلامت روانى \\
\hline \multirow[t]{2}{*}{$\cdot / \Delta \mu$} & $.1 . .1$ & $|r / r . \pm r / q|$ & $\mid N / K \cdot \pm 1 / r q$ & يس آزمون \\
\hline & & & \multicolumn{2}{|r|}{ عملكرد اجتماعى } \\
\hline .1 .9 & . TS & $\mid r / q \mu_{ \pm} \mu / \Delta V \pm$ & $\| / K \varepsilon \pm r / \varepsilon F$ & ي يِشآزمون \\
\hline \multirow[t]{2}{*}{.$/ 4 \lambda$} & $.1 . .1$ & $\mid \mu / \mu \mu \pm \Psi / \| \pm \pm$ & $r \cdot / 1 \cdot \pm r / I I$ & يسآزمون \\
\hline & & & & \\
\hline .4. & •/V\& & $\mid r / \mu \mu \pm r / s 9$ & 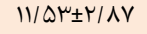 & 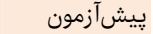 \\
\hline \multirow[t]{2}{*}{.$/ r k$} &.$/ .1$ & $\Delta / V \mu^{\mu} \pm 1 / 4 \lambda$ & $V / \Delta \mu_{ \pm} \pm / / \mu \mid$ & يسآزمون \\
\hline & & & & سلامت عمومى \\
\hline $.1 \cdot V$ & . ITA & $\mid \Psi / r \cdot \pm r / \Delta G$ & $11 / k 9 \pm r / 19$ & 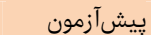 \\
\hline \multirow[t]{2}{*}{.$/ 48$} & $.1 . .1$ & $|r / F| \pm Y / 9 q$ & $\mid V / \Lambda \varsigma \pm I / K Q$ & يֶسآزمون \\
\hline & & & & كيفيت زندكى \\
\hline.$/ . V$ & - VQ & $\Delta V / \cdot \bullet \pm 11 / \cdot 9$ & $0.199 \pm 1 . / \Delta 9$ & بيش آزمون \\
\hline$\cdot \mid \Delta \Lambda$ & $.1 . .1$ & $\varepsilon N / \mu \mu_{ \pm} \cdot 1 \cdot / \mu$ & 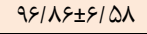 & 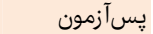 \\
\hline
\end{tabular}

بحث

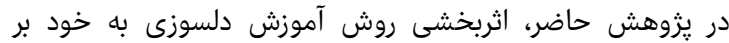

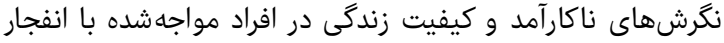

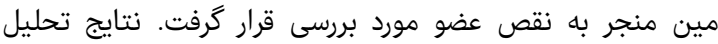

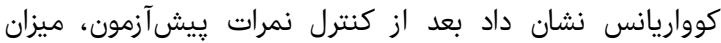

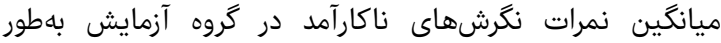

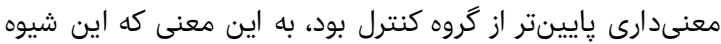

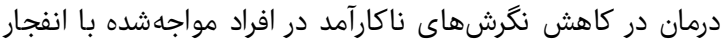

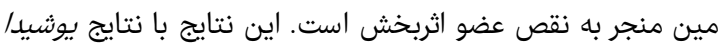

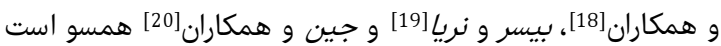

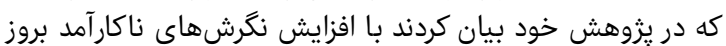

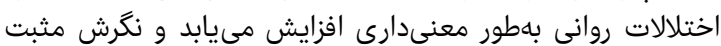
مرتبط با تعديل نشانههاى علايم رِآسي آسيبى است.

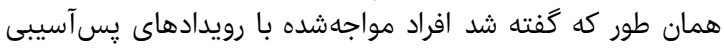

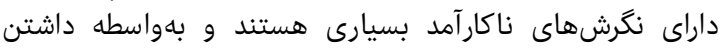

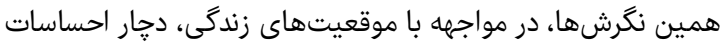

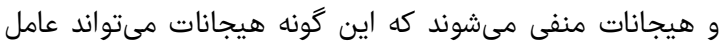

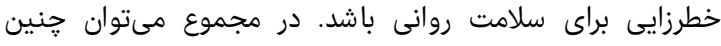

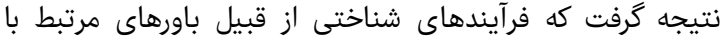

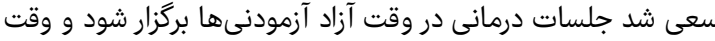

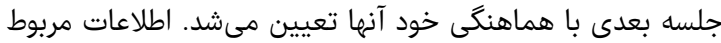

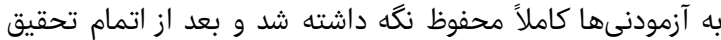

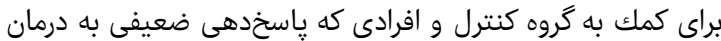

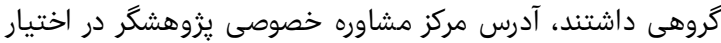

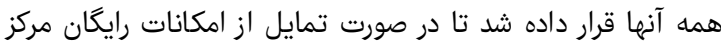

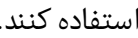

جدول () خلاصه محتواى جلسات آموزش متمركز بر دلسوزى جلسه اول تروما و ترول تعريف تروما و انواع آن و اثرات رويداد آسيبزاى انفجار مين منجر به نقص

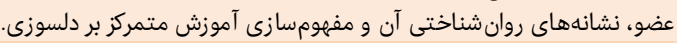

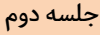

آموزش همدلى: آموزش براى درك و فهم اينكه افراد احساس كنند كه امور را با نكَرش همدلانه دنبال كنند.

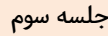

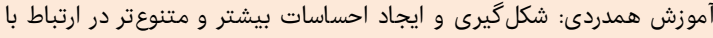
مسايل افراد براى افزايش مراقبت و وتوجه بـ به سلامتى خود. جلسه جهارم إنراد براي

آموزش بخشايش: يذيرش اشتباهات و بخشيدن خود بهخاطر اشتباهات براى سرعتبخشيدن به ايجاد تغييرات.

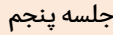

آموزش يذيرش مسايل: يذيرش تغييرات بيش رون رو و و تحمل شرايط سخت و

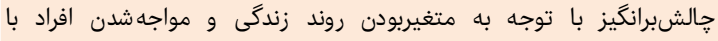
جالشهاى مختلف.

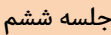

آموزش رشد احساسات ارزشمند و متعالى: ايجاد احساسات ارزشمند در خود تا باستا

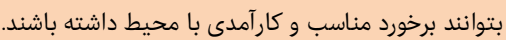
جلسه هفتم

آموزش مسئوليتيذيرى: آموزش مسئوليتيذيرى مولفه اساسى آموزش براس

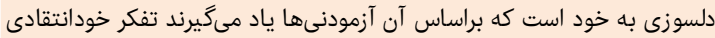

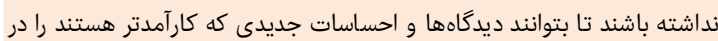

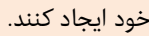
جلسه هشتم

آموزش و تمرين مهارتها: مرور و تمرين مهارتهاى ارايهشده در جلسات

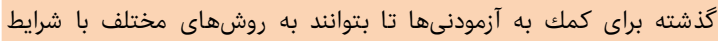
متفاوت زندگى خود مقابله كنند.

در پايان، دادههاى جمعآورىشده با استفاده از آزمون تحليل

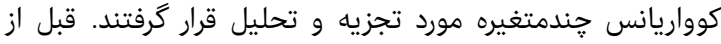

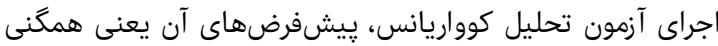

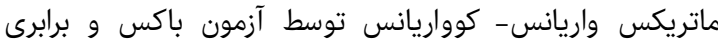
واريانسهاى دو گروه توسط آزمون لوين بررسى شدان.

يافتهها

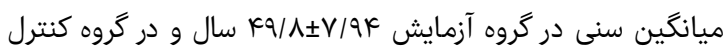
ابتدائ N/QH

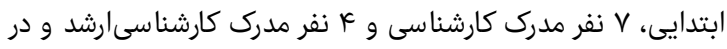

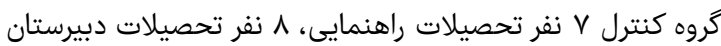
و و ه نفر مدرك كارشناسى داشتند.

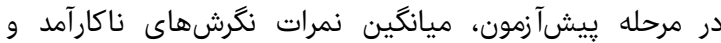

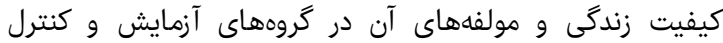

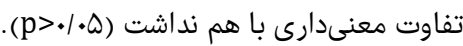

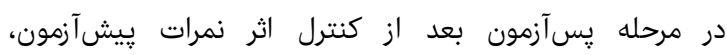

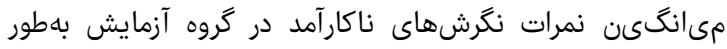

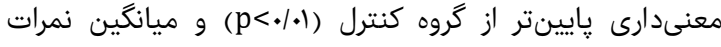




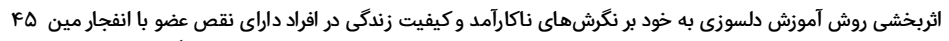

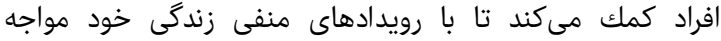

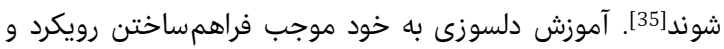

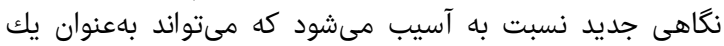

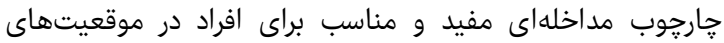

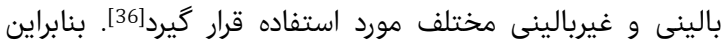

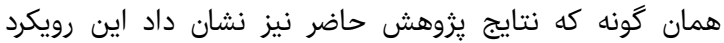

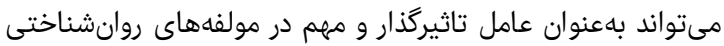
مثبت انسانها تلقى شود.

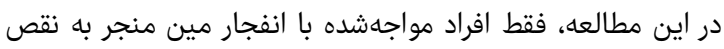

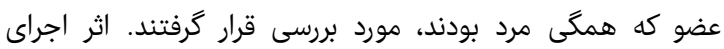

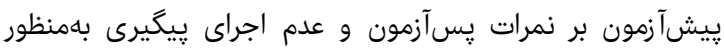

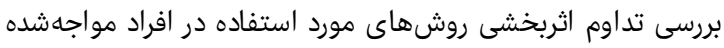

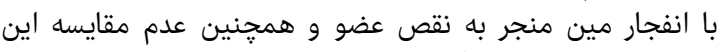

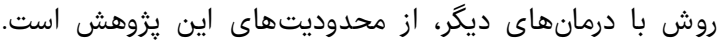

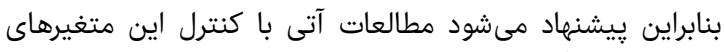

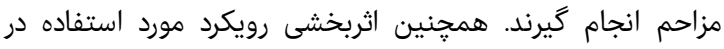

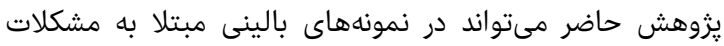
روانشناختى مختلف مورد بررسى قرار گيرد.

نتيجه

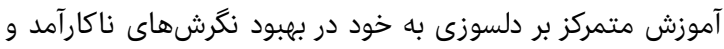

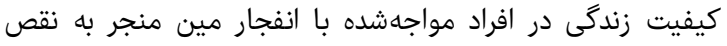

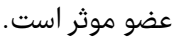

تشكر و قدردانى: از همكارى و همراهى صميمانه بنياد شهيد

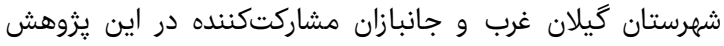

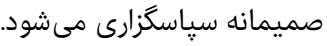

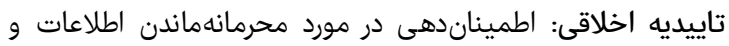

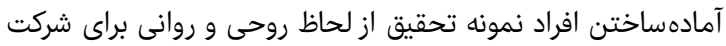

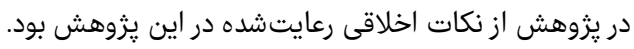

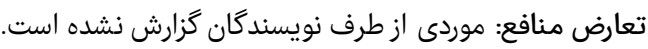

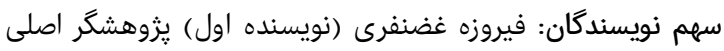

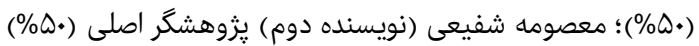

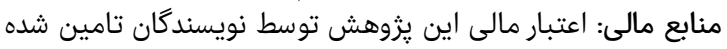
است.

منابع

1- Başoğlu M, Livanou M, Salcioğlu E, Kalender D. A brief behavioral treatment of chronic post traumatic stress disorder in earthquake survivors: Results from an open clinical trial. Psychol Med. 2003;33(4):647-54.

2- Abhari-Ahmadi SA. Long-term effects of war on psychosocial status of patients with post-traumatic stress disorder. Proceedings of The Third Symposium of Neurological Complications Psychol War. Tehran: Golban Press; 2000. pp. 83-7. [Persian]

3- Yavari A, Esfandiari E, Masoumi M, Saeedi H, Allami M. Consideration of chronic pain and current prosthetic satisfaction. in above knee amputees. Iran J War Public Health. 2011;3(10):53-8. [Persian]

4- Seyed Hosseini Davarani SH, Moosavi B, Karbalaei Esmaeili S, Soroosh MR, Masoumi M. Service satisfaction among war related bilateral lower limb amputation Iran J War Public Health. 2010;3(1):49-54. [Persian]

5- Azarmi S, Farsi Z, Sajadi S.A. Development of adaptation questionnaire using roy's adaptation model and its psychometrics on veterans with lower limb amputation. Hayat. 2013;19(4):26-37. [Persian]

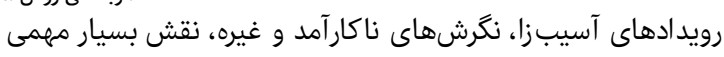

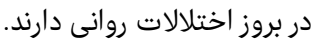

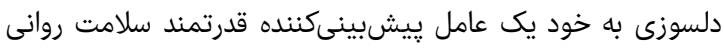

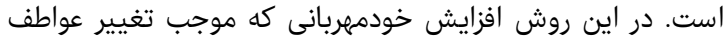

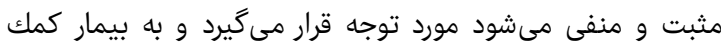

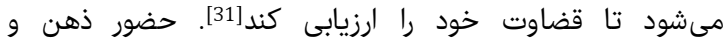

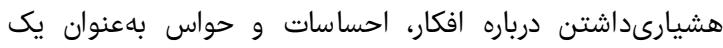

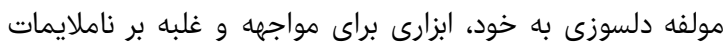

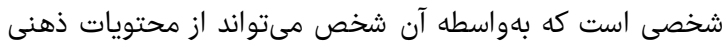

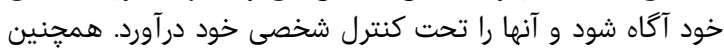

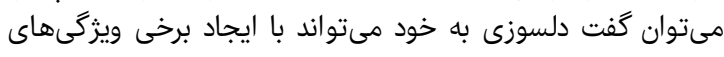

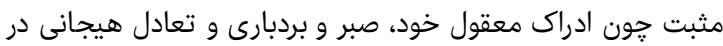

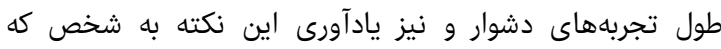

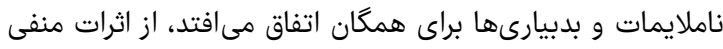

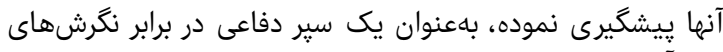

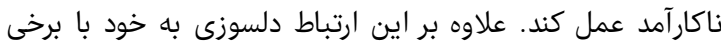

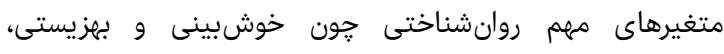

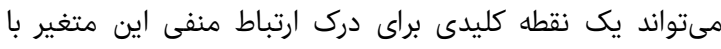

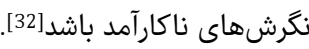

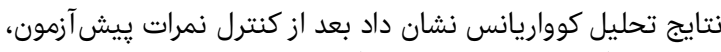

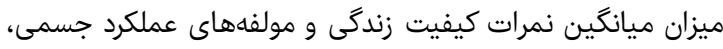

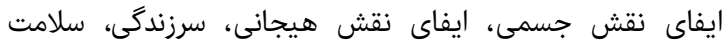

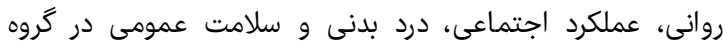

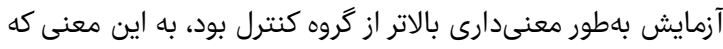

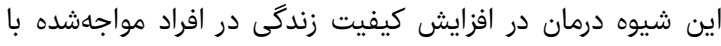

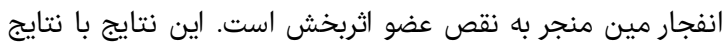

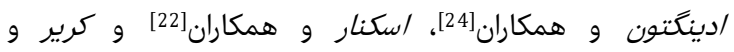

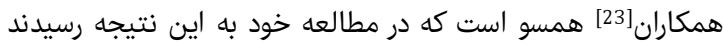

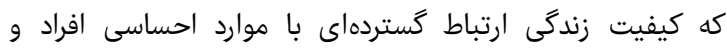

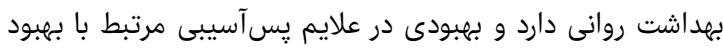

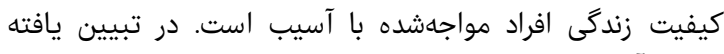

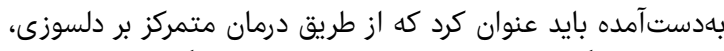

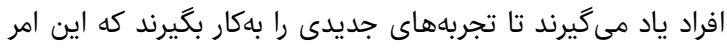

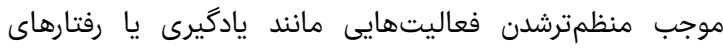

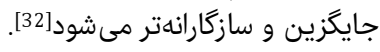

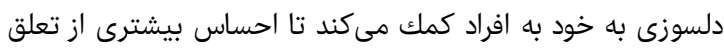

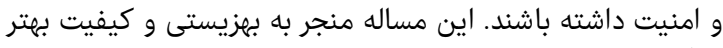

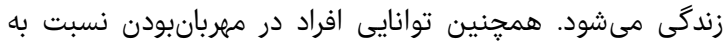

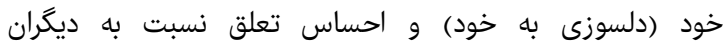

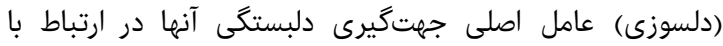

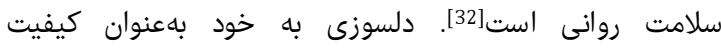

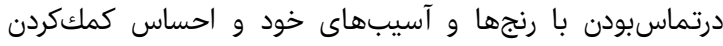

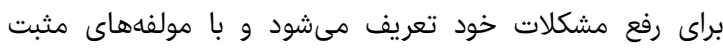

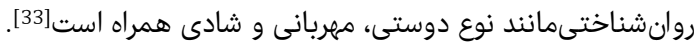

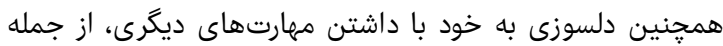

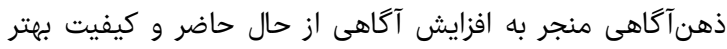

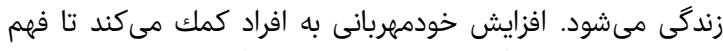

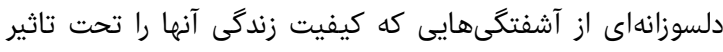

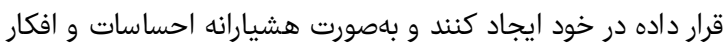

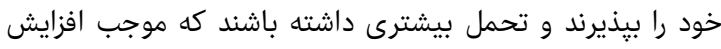

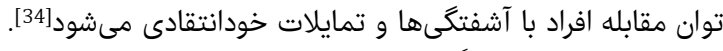

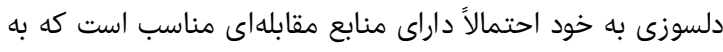


Dehkardi B. quality of life and Quality of life and its influencing factors in breast cancer patients unde rgoing chemotherapy. Epidemiol Iran. 2007;3(3-4):61-6. [Persian]

22- Schnurr PP, Lunney CA. Symptom benchmarks of improved quality of life in PTSD. Depress Anxiety. 2016;33(3):247-55.

23- Currier JM, Drescher KD, Hollan JM, Lisman R, Foy DW. Spirituality, forgiveness, and quality of life: Testing a mediational model with military veterans with PTSD. Int J Psychol Relig. 2016;26(2):167-79.

24- Mandani B, Fakhri A. Study of health related quality of life in posttraumatic stress disorder war veterans. Iran J War Public Health. 2013;5(2):18-25. [Persian]

25- Gilbert P, Procter S. Compassionate mind training for people with high sham eand selfcriticism: A pilot study of a gr oup therapy approach. Clin Psychol Psychother. 2006;13(6):352-79.

26- Ebrahimi A, Neshatdoost HT, Kalantari M, Molavi H, Asadollahi Gh. Contributions of dysfunctional attitude scale and general health subscales to prediction and odds ratio of depression. J Shahrekord Univ Med Sci. 2008;9(4):52-8. [Persian]

27- Beck AT, Brown G, Steer RA, Weissman AN. Factor analysis of the dysfunctional attitude scale in a clinical population. Psychol Assess. 1991;3(3):478-83.

28- Bayanzadeh SA, Bolhary J, Dadfar M, Karimi Keisomi I. Effectiveness of cognitive-behavioral religious cultural therapy in improvement of obsessivecompulsive patients. Razi J Med Sci. 2005;11(44):913-23. [Persian]

29- Rostami R, Moghadasetabrizi Y, Dar veeshpour KA, Montazeri A. The effect of exercise on quality of life of opioid dependent patients under methadone maintenance therapy. Appl Psychol Res Q. 2012;3(1):111. [Persian]

30- Gilbert P. Introducing comp-assion- focused therapy. Adv Psychiatri Treat. 2009;15(3):199-208.

31- Barnard LK, Curry JF. Self-Compassion: Conceptualizations, correlates \& int rventions review of general psychology. Rev Gen Psychol. 2011;15(4):289303.

32- Raque-Bogdan TL, Ericson SK, Jackson J, Martin HM, Bryan NA. Attachment and mental and physical health: Self-compassion and mattering as medi ators. J Couns Psychol. 2011;58(2):272-8.

33- Germer CK, Neff KD. Self-compassion inclinicalpractice. J Clin Psychol. 2013;69(8):2-12.

34- Neff KD, Hseih Y, Dejitthirat K. Self -compassion, achievement goals, and cop ing with academic failure. J Psychol Educ. 2005; 4(3):263-87.

35- Allen BA, Leary MR. Self-Compassion, Stress and Coping. Soc Personal Psychol Compass. 2010;4(2):107-18. 36- Reddy SD, Negi LT, Dodson-Lavelle B, Ozawa-de Silva B, Pace TW, Cole S P, et al. Cognitive-based compassion training: A promising prevention strategy for at-risk adolescents. J Child Fam Stud. 2013;22(2):219-30.
6- American Psychiatric Association. Diagnosis and statistical manual disorder. $4^{\text {th }}$ edition. Washington DC: American Psychiatric Association; 2000. pp. 165-73.

7- Raes F. Rumination and worry as mediators of the relationship between self-compassion and depression and anx iety. Personal Individ Differ. 2010;48(6):757-61. 8- Terry LM, Leary RM. Self-compassion, self-regulation and health. J Self Identit. 2011;10(3):352-62.

9- Seligowski VA, Miron RL, Orcutt KH. Relations among self-compassion, PTSD symptoms, and psychological health in a trauma-exposed sample. Mindfulness; 2015;6(5):1033-41.

10- Ehreta MA, Joormannb J, Berkingc M. Examining risk and resilience factors for depression: The role of selfcriticism and self-compassion. Cogn Emot. 2015;29(8):1496-504.

11- Zeller M1, Yuval K, Nitzan-Assayag Y, Bernstein A. Self-compassion in recovery following potentially traumatic stress: Longitudinal study of at-risk youth. J Abnorm Child Psychol. 2015;43(4):645-53.

12- Miron RL, Sherrill AM, Orcutt KH. Fear of selfcompassion and psychological inflexbility interact to predict PTSD symptom severity. J Contextual Behav Sci. 2015;4(1):37-41.

13- Woodruff CS, Glass RC, Arnkoff BD, Crowley JK, Hindman KB, Hirschhorn WE. Comparing selfcompassion, mindfulness, and psychological inflexibility as predictors of psychological health. Mindfulness. 2014;5(4):410-21.

14- de Graaf LE, Roelofs J, Huibers MJ. Measuring dysfunctional attitudes in the general population: The dysfunctional attitude scale (form A) revised. Cognit Ther Res. 2009;33(4):345-55.

15- Weich S, Churchill R, Lewis G. Dysfunctional attitudes and the common mental disorders in primary care. J Affect Disord. 2003;75(2):269-78.

16- Lewinshon PM, Joiner TE, Rhode P. Evaluation of cognitive diathesis stress models in predicting major depressive disorder in adolescents. J Abnorm Psychol. 2001;110(2):203-15.

17- Ellis A. How may therapy and Practice of psychotherapy has influenced and changed other psychotherapies. J Ration Cogn Ther. 2004;22(2):79-83.

18- Yoshida H, Kobayashi N, Honda N, Matsuoka H, Yamaguchi T, Homma H, et al. Post-traumatic growth of children affected by the Great East Japan Earthquake and their attitudes to memorial services and media coverage. Psychiatry Clin Neurosci. 2016;70(5):193-201.

19- Besser A, Neria y. PTSD symptoms, satisfaction with life, and prejudicial attitudes toward the adversary among Israeli civilians exposed to ongoing missile attacks. J Trauma Stress. 2016;22(4):268-75.

20- Jain S, McLean C, Adler EP, Rosen CS. Peer support and outcome for veterans with posttraumatic stress disorder (PTSD) in a residential rehabilitation program. Community Ment Health J. 2016;52(8):1089-92.

21- Safaee A, Zeykhami B, Tabatabaee SH, Moghimi 